\title{
La estrategia del Population Council: Promoción de transiciones a la vida adulta sanas, seguras y productivas
}

Population Council

Follow this and additional works at: https://knowledgecommons.popcouncil.org/departments_sbsr-pgy

Part of the Demography, Population, and Ecology Commons, Family, Life Course, and Society Commons, Gender and Sexuality Commons, International Public Health Commons, and the Medicine and Health Commons How does access to this work benefit you? Let us know!

\section{Recommended Citation}

"La estrategia del Population Council: Promoción de transiciones a la vida adulta sanas, seguras y productivas," Promoción de transiciones a la vida adulta sanas, seguras y productivas. New York: Population Council, 2011. 


\section{El desafío}

\section{La adolescencia es una etapa crítica del desar- rollo humano durante la cual se sientan las bases para la vida adulta.}

$\mathrm{M}$ ás de 1,5 mil millones de personas de entre 10 y 24 años en países en vías de desarrollo se encuentran en la transición entre la niñez y los roles y responsabilidades de la edad adulta -como trabajador, ciudadano, cónyuge y padreen un período de cambios sin precedentes a nivel internacional. Las próximas generaciones de jóvenes serán aún más grandes. Sin la adecuada inversión en su salud y educación y en oportunidades para que desarrollen medios de vida productivos, las perspectivas de futuro de los jóvenes serán limitadas. Los jóvenes afrontan desafíos particulares en África, donde la mitad de las nuevas infecciones por VIH ocurren en personas de entre 15 y 24 años de edad y donde las jóvenes mujeres son quienes se contagian principalmente. Los futuros de muchas naciones, así como el logro de muchos de los Objetivos de Desarrollo del Milenio de la ONU, dependen de cuán eficazmente los adolescentes atraviesen esta etapa de sus vidas.

\section{La estrategia del Population Council}

El enfoque del Population Council en los adolescentes comenzó a principios de 1990 y estuvo acompañado del compromiso de hacer que el ámbito de la investigación y los programas para adolescentes cambie de un enfoque en la sexualidad, la salud

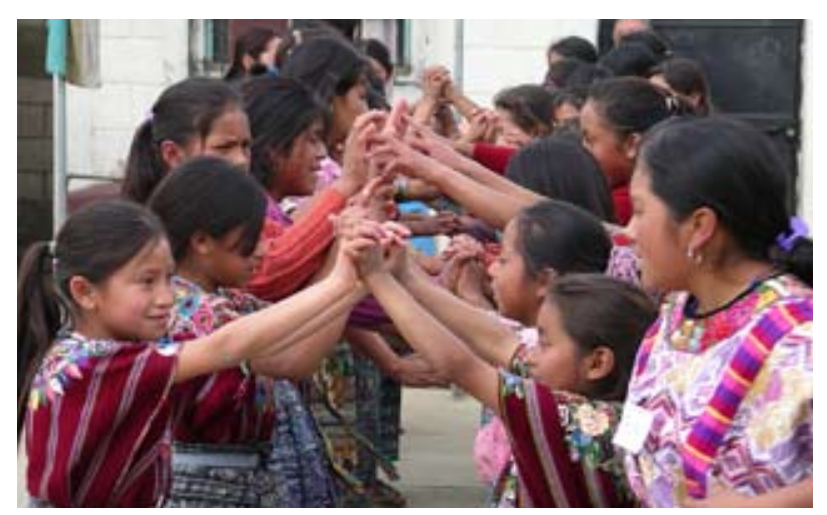

El programa Abriendo Oportunidades crea espacios seguros para las niñas mayas y les brinda mentores.

reproductiva y el comportamiento a uno que contemple los problemas sociales y económicos más amplios que constituyen la base de la salud y el bienestar de los adolescentes.

En 1998, se lanzó el actual programa de trabajo con la publicación de The Uncharted Passage -una revisión de la situación de los jóvenes adolescentes en países en vías de desarrollo que sirve como punto de referencia-. La estrategia del Population Council ha tenido cuatro características fundamentales:

\section{Atención constante a las implicancias del} género durante la adolescencia, en especial, las necesidades de las niñas en los países menos desarrollados. En estos entornos, las oportunidades de las niñas generalmente están limitadas; con frecuencia experimentan violencia de género, pueden correr el riesgo de ser sometidas a casamientos acordados y se ven afectadas de manera desproporcionada por la epidemia de VIH.

- Una estrategia multisectorial, que compromete diferentes facetas de la sociedad que ejercen influencia sobre las vidas de las niñas adolescentes: familias y líderes comunitarios; niños y hombres; organizaciones no gubernamentales y comunitarias; y los sectores de la salud y la educación. La poca salud en los adolescentes no está generada por factores fisiológicos solamente, sino que está determinada por el aislamiento y la vulnerabilidad económica de los jóvenes, en especial las mujeres.

- Una preocupación especial ante la desigualdad, según el género, la clase social y económica, el lugar de residencia urbano/rural, y el acceso a la economía moderna, que se intensifica durante la adolescencia.

- Un compromiso con la investigación de políticas basada en las evidencias, combinada con el desarrollo y la evaluación de programas, lo que garantiza que se apliquen rápidamente los resultados de los análisis de políticas en los programas y las políticas reales. 


\section{Teoría del cambio}

La teoría del cambio del Population Council sostiene que la base de una vida productiva -que puede implicar el matrimonio, nacimiento de hijos, empleo, ciudadanía y suficiencia económica - se forma durante la adolescencia. Entre los elementos fundamentales de esta base se encuentran las habilidades para la vida cotidiana, los conocimientos sobre finanzas, la salud sexual y reproductiva, las habilidades de negociación y la alfabetización. Este también es el período durante el cual la pobreza se puede consolidar o superar $y$, frecuentemente, cuando la violencia de género se afronta por primera vez. De modo que nuestro enfoque es crear valores sociales y económicos para los adolescentes de las poblaciones más vulnerables, a fin de garantizar una transición segura y productiva a los roles de adulto. Una vez que los valores se adquieren, una persona joven no los pierde $y$, aunque los beneficios de estos valores pueden no plasmarse completamente hasta llegar a la edad adulta (por lo general algunos años después de alcanzarla), sí es posible evaluar resultados parciales.

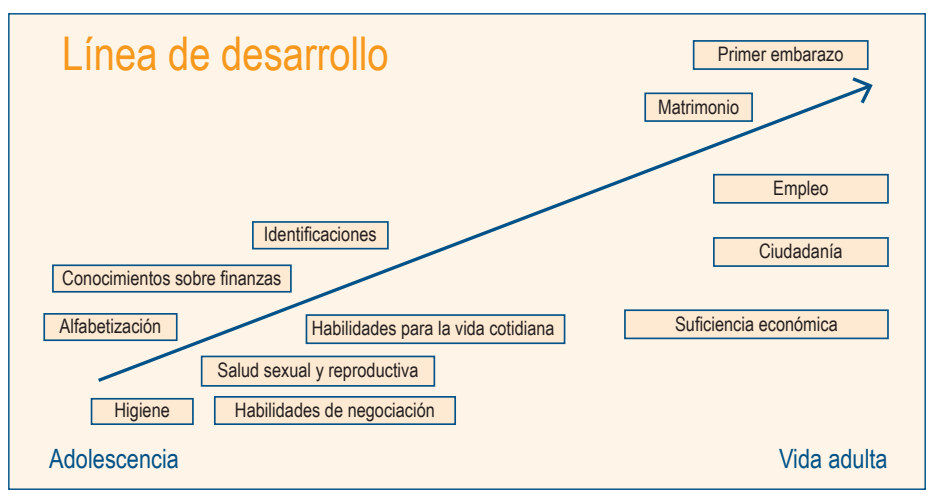

\section{Estrategia}

El Population Council usa información para comprender mejor la diversidad de vidas y experiencias de los adolescentes y para identificar a los más vulnerables y desatendidos. Luego, trabaja con socios locales para desarrollar programas que desarrollen valores para estos adolescentes, mide los desafíos que ocurren a nivel individual y comunitario, y facilita la ampliación de las estrategias exitosas. Mientras se avanza con la estrategia, se desarrollan herramientas de investigación y programas que pueden compartirse en el ámbito de acción. El compromiso con los formuladores de políticas - a nivel comunitario, nacional e internacional- está presente en cada etapa del trabajo y garantiza que se desarrollen políticas para abordar las necesidades y experiencias de este grupo crítico de la población.

\section{Recolección, análisis y divulgación de las evidencias sobre la} diversidad de vidas y experiencias de los adolescentes

El Population Council ha estipulado como prioridad obtener los datos existentes y recolectar nuevos datos para comprender las distintas influencias de edad, género, escolaridad, estado civil, organización en viviendas y residencias urbanas y rurales en las experiencias de los adolescentes.

El análisis secundario de las nóminas de hogares de las Encuestas Demográficas y de Salud brinda información básica sobre los adolescentes que viven en esos hogares. Esta información se resume en 55 guías específicas por cada país creadas por el Population Council: La experiencia de los adolescentes en profundidad: uso de datos para identificar y llegar a los jóvenes más vulnerables. El propósito de esta serie es brindar a los encargados de la toma de decisiones de todos los niveles - desde gobiernos, organizaciones no gubernamentales (ONG) y grupos de apoyo- la información sobre la situación de los adolescentes y las jóvenes mujeres. El rango de edad cubierto es de 10 a 24 años. La información se presenta en gráficos, tablas y mapas (siempre que sea posible), en múltiples formatos para que los datos sean accesibles para diferentes tipos de audiencias (TA N. ${ }^{\circ} 10$ ).

Encuestas representativas a nivel nacional y subnacional. Los datos existentes rara vez brindan información en profundidad obtenida de entrevistas con los adolescentes. El Population Council ha implementado encuestas en todo el mundo para obtener información sobre los estilos de vida de los adolescentes. Se condujeron encuestas nacionales en Bangladesh, Egipto, Etiopía, India y Pakistán, y las encuestas Iongitudinales realizadas en Malawi y Sudáfrica brindan la oportunidad única de realizar un seguimiento de los adolescentes en el tiempo.

Presentación de la información de manera tal que sea fácil de usar Una gran cantidad de datos que se recolectan en el mundo nunca se analizan. Incluso los que se analizan, frecuentemente se presentan de una manera que solo puede ser interpretada por estadísticos profesionales. El Population Council se esfuerza por lograr que los datos sean accesibles para diferentes audiencias, a fin de llevar a cabo la planificación, implementación y evaluación del programa, y analizar las políticas. Además de crear mapas a partir de estadísticas, usamos cada vez más el desarrollo de mapas como ejercicio de participación, en el cual los adolescentes de las comunidades locales delinean y describen el contexto de sus vidas, definen problemas, proponen mecanismos para afrontar situaciones y diseñan estrategias de desarrollo ( $T A N^{\circ} .{ }^{\circ} 30$ ).

\section{Creación de herramientas innovadoras para el ámbito de acción}

En su análisis de datos e implementación de programas, el Population Council crea recursos y herramientas innovadoras para que otras personas usen. Una herramienta importante para recolectar información de los adolescentes sobre temas delicados ha sido la autoentrevista de audio asistida por computadora (ACASI, por sus siglas en inglés). El Population Council desarrolló su propio software para esta herramienta, con lo que realzó aún más la habilidad de garantizar la estandarización, la confidencialidad, la flexibilidad lingüística y la seguridad en la recolección de los datos.

El trabajo de gran alcance que realizamos para el desarrollo de programas, sumado a nuestras intervenciones, ha dado como resultado dos recursos principales:

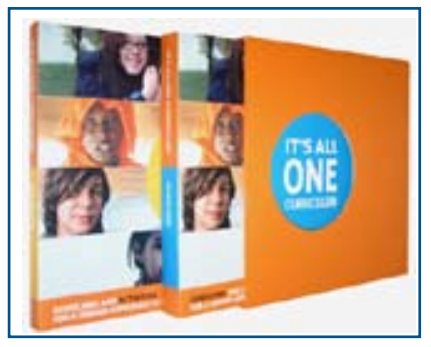

It's All One Curriculum (Un sólo currículo), un plan de estudio de dos volúmenes que ubica las cuestiones de género y los derechos humanos en el centro de la educación en sexualidad y VIH, con 54 actividades que involucran a los jóvenes $y$ fomentan habilidades de pensamiento crítico. Disponible en inglés, español y francés. www.itsallone.org 


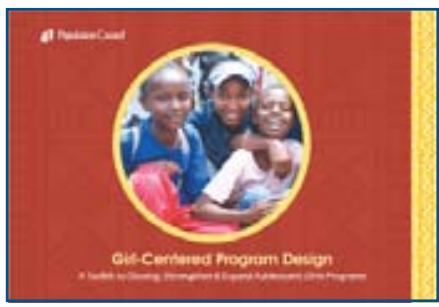

Diseño de programa centrado

en las niñas: un conjunto de

herramientas para desarrollar, fortalecer y ampliar los programas para niñas adolescentes. Este conjunto de herramientas se centra en la estructura, el contenido y la evaluación de los programas para niñas. Es una herramienta práctica para comenzar o fortalecer programas de niñas y está disponible en inglés, español y francés. www.popcouncil.org/publications/books/2010_ AdolGirlsToolkit.asp

\section{Uso de espacios seguros para construir valores y gozar de una vida adulta saludable y productiva}

El alcance y la cobertura limitada de los programas existentes para jóvenes y su enfoque en la información y los servicios de salud no han abordado lo suficientemente las necesidades de muchos adolescentes. Estos jóvenes pueden estar fuera del sistema escolar, lejos de su hogar, pueden ser casados o estar atrapados en un ciclo de explotación laboral y pobreza. El Population Council concentró sus esfuerzos iniciales en la creación de valores para las niñas adolescentes, quienes deben afrontar riesgos desproporcionados. Las intervenciones experimentales se diseñaron conjuntamente con socios locales para ofrecer a las adolescentes protegidas espacios de reunión. En estos "lugares seguros", las niñas desarrollan relaciones saludables con pares y mentores, ganan confianza, adquieren conocimientos y aprenden nuevas habilidades.

\section{Lecciones aprendidas}

\section{¿A quiénes llegamos?}

Las intervenciones del Population Council han demostrado que es posible llegar a los adolescentes vulnerables que no han sido incluidos en las inversiones en jóvenes. Mediante estrategias de reclutamiento dirigidas, estas intervenciones han identificado y reclutado poblaciones objetivo.

- Las niñas en riesgo de contraer matrimonio a una temprana edad son generalmente quitadas de la atención de la gente, pero fueron reclutadas en intervenciones culturalmente aceptables en Bangladesh, Burkina Faso, Egipto, Etiopía, Guatemala, India y Kenia, y se logró reunir evidencias de que, como resultado de estas intervenciones, aumentó significativamente la edad en la que contrajeron matrimonio (TA N. ${ }^{0} 13 ; 9 ; 12 ; 20 ; 5 ; 2 ; 19 ; 29$ ).

- Las niñas casadas son el grupo al cual es más difícil llegar. Después del casamiento, las niñas generalmente se mudan al hogar de la familia del esposo, un lugar en el que tienen conexiones sociales muy limitadas y que comparten con un hombre que es, por lo general, mucho mayor que ellas. Los proyectos de Burkina Faso, Etiopía e India lograron que estas niñas salieran de las penumbras y las conectaron entre sí para que obtengan apoyo social y acceso a servicios (TA N. ${ }^{\circ}$ 9; 20; 8). El proyecto First Time Parents (Padres por primera vez), en India, comprometió a madres y padres y se enfocó en los aspectos positivos de ser padres por primera vez, reevaluó los roles de género y las expectativas en torno al cuidado de los niños, la preparación de alimentos, la búsqueda de agua, etc.
- Los trabajadores domésticos de los hogares urbanos pobres experimentan condiciones de trabajo calamitosas, reciben salarios bajos, tienen horarios de trabajo agotadores y, con frecuencia, son víctimas de abuso sexual. En Etiopía, donde muchas niñas de zonas rurales llegan a Addis escapando de quienes quieren que se casen a una temprana edad, se identificaron trabajadores domésticos con una estrategia de reclutamiento puerta a puerta. Las negociaciones con los empleados dieron como resultado la creación de un programa de espacios seguros, Biruh Tesfa (TA N. ${ }^{\circ} 21$ ): en un edificio del gobierno en los barrios marginales de Addis, las niñas de entre 10 y 19 años de edad se reúnen todas las semanas con mentores a fin de adquirir valores sociales y económicos para reconstruir sus vidas.

- Jóvenes adolescentes. La primera ola de intervenciones estuvo orientada a niñas de 150 más años de edad. Pero pronto quedó claro que era necesario intervenir adecuadamente antes de que las niñas lleguen a ciertos eventos (matrimonio a temprana edad, abandono de la escolaridad, relaciones sexuales por primera vez, etc). Cada vez más proyectos están llegando a niñas de entre 10 y 14 años.

Niñas en riesgo de contraer $\mathrm{VIH}$. Las adolescentes continúan siendo las más afectadas por las infecciones con VIH. Los programas de espacios seguros crean relaciones positivas con los pares, habilidades de negociación y valores económicos que pueden ayudar a las niñas a evitar conductas no deseadas y/o riesgosas.

\section{¿Qué proveemos?}

Además de los valores sociales que se crean en los espacios seguros, muchas de las intervenciones se concentran en construir capacidades financieras, ahorros, oportunidades de trabajo y salud sexual y reproductiva:

- Siyakha Nentsha, en Sudáfrica, aprovecha las altas tasas de inscripción en las escuelas, en KwaZulu Natal, y trabaja con 1.100 niñas y niños en sus escuelas. Un componente del programa son los conocimientos sobre finanzas, y el plan de estudio ha sido certificado por el gobierno, de modo que la compleción del programa brinda importantes acreditaciones a los jóvenes (TA N. $\left.{ }^{\circ} 4\right)$.

- Al trabajar a través de agrupaciones de adolescentes en zonas rurales y escuelas técnicas no formales en zonas urbanas, el programa Kishori Abhijan, en Bangladesh, llega a niñas de entre 15 y 19 años de edad para promocionar la educación en finanzas mediante el uso de mentores. Estamos evaluando el valor de agregar mentores a las estrategias educativas tradicionales en materia de finanzas (TA N. ${ }^{\circ} 13$ ).

- Safe and Smart Savings (Ahorros seguros e inteligentes) es una ampliación de uno de los primeros programas de espacios seguros en Kenia, Binti Pamoja. Ubicado en el barrio marginal de Kibera, el programa va más allá de la educación en finanzas para desarrollar un programa bancario en asociación con dos bancos de Nairobi. Actualmente, el programa se está ampliando a Uganda. Llega a 2.300 jóvenes, pero cuando alcance la fase de lanzamiento completo, llegará a más de 20.000 (TA N. $\left.{ }^{\circ} 29\right)$.

\section{¿Cómo se vinculan estos programas con otros servicios?}

Las intervenciones no proveen servicios de salud directamente, sino que relacionan a los participantes con los servicios existentes en la comunidad 
a través de visitas de exposición, colaboraciones formales y campañas de concientización.

\section{- Medios de vida/ahorros. En Kenia, el programa se asocia con bancos} comerciales para garantizar que las niñas tengan acceso a los servicios del sector financiero privado (TA N. ${ }^{\circ} 29$ ). En Sudáfrica, el plan de estudio brinda conocimientos a los adolescentes acerca de los programas de asistencia financiera del gobierno, para los cuales muchos reúnen los requisitos, pero muy pocos saben cómo acceder.

- Educación. El programa Ishraq, en Egipto, es un programa de "segunda oportunidad" cuyo objetivo es llegar a niñas que han abandonado la escolaridad y orientarlas hacia escuelas del gobierno al final del programa. Al final del proyecto piloto, el $92 \%$ de las niñas que hicieron el examen lo aprobaron y se inscribieron en el sistema escolar formal (TA N. ${ }^{0}$ 12).

Servicios para la violencia de género. Aunque no se desarrolló como programa de violencia de género desde el principio, los mentores de los programas de espacio seguro observaron que el tema de la violencia emergía como una cuestión principal en todos los lugares de intervención. Los espacios para niñas solamente se convirtieron en un lugar seguro para entablar estos debates, aprender acerca de sus derechos y compartir estrategias para afrontar la situación. Estos programas evolucionan continuamente como lugares para la prevención de la violencia.

\section{¿Pueden estos programas llevarse a escala?}

La ampliación de un programa depende en gran medida de la existencia de un gobierno, ONG u organizaciones comunitarias y basadas en la fe, con la capacidad de implementar el programa dentro de una población mayor. El modelo para esta ampliación difiere significativamente según los contextos, pero las siguientes son algunas características comunes: 1) establecimiento de relaciones con posibles socios de ampliación y 2) atención al costo del programa y simplificación de este.

- El proyecto Biruh Tesfa, en los barrios marginales de Etiopía, se asoció con el gobierno desde el inicio del programa. Trabajando desde centros sociales donados por el kebele, o gobierno local, el programa ahora llega a 16.500 niñas que están fuera del sistema escolar, y se está expandiendo a otras 12 ciudades para llegar a otras 30.000 niñas. Los salarios de los mentores también se fijaron en concordancia con el salario de un trabajador social del gobierno, con lo que se garantiza la posibilidad de ampliar el programa con recursos del gobierno (TA N. ${ }^{0} 21$ ).

- En las zonas rurales de Alto Egipto, el programa Ishraq trabaja con 1.800 niñas de entre 12 y 15 años de edad para fomentar la asistencia a escuelas e incrementar los conocimientos sobre salud. El programa ya obtenido un fuerte apoyo de la comunidad y el gobierno, se encuentra en una fase de expansión y se espera que se convierta en un programa patrocinado por el gobierno en el futuro cercano (TA N. $\left.{ }^{0} 12\right)$.
- El programa Abriendo Oportunidades, en Guatemala, llega a 1.260 niñas mayas de zonas rurales de entre 8 y 18 años. Los programas se llevan a cabo en centros sociales y escuelas, y emplean un modelo de mentores que asumen los roles en forma gradual, de manera que las niñas pueden avanzar hacia posiciones de liderazgo en el programa. Los gobiernos locales, las ONG y el sector comercial privado están cada vez más comprometidos y brindan los lazos necesarios para la sostenibilidad y la institucionalización. Además de la ampliación del programa en sí mismo, el modelo de Abriendo está siendo adoptado y replicado por el público, las ONG y los socios del sector privado de Guatemala, y está a punto de convertirse en el primer programa nacional para niñas aborígenes de la región (TA N. $\left.{ }^{\circ} 5\right)$.

- El programa Siyakha Nentsha, en las escuelas de Sudáfrica, ya es un plan de estudio acreditado y el Ministerio de Educación está ansioso por ampliar el programa en toda KwaZulu-Natal y más allá de sus fronteras (TA N. ${ }^{\circ} 4$ ).

- El programa Berhane Hewan, en la zona rural de Etiopía, ha llegado a 12.000 niñas casadas y solteras de entre 10 y 19 años en una región en la que la mitad de las niñas ya están casadas a los 15 años. Las estructuras del kebele en los pueblos ofrecen el lugar y, con el apoyo del gobierno, el programa llegará a 72.000 niñas (TA N. ${ }^{\circ} 20$ ).

- El programa Kishori Abhijan tiene como objetivo reducir las tasas de abandono de la escuela, incrementar la actividad económica independiente de las niñas y aumentar la edad en la que se casan. El programa es un esfuerzo conjunto del Population Council, UNICEF, UNFPA, el Instituto de Estudios de Desarrollo de Bangladesh (BIDS), el Comité de Desarrollo Rural de Bangladesh (BRAC) y el Centro para la Educación Masiva en Ciencias (CMES) y combina habilidades para la vida cotidiana y capacitación en medios de vida con el BRAC and el CMES, grandes y reconocidas ONG que han implementado el programa en 14 distritos rurales, con la inscripción de 15.000 niñas de entre 13 y 22 años. Según una prueba piloto exitosa evaluada por el Population Council y el BIDS, el componente de habilidades para la vida cotidiana se está ampliando a través del BRAC y el CMES con el fin de inscribir a más de 250.000 niñas en 58 distritos (TAN. ${ }^{\circ}$ 13).

\section{¿Cuál es el siguiente paso?}

La próxima etapa de este trabajo es demostrar empíricamente de qué manera las inversiones en habilidades fundamentales para la vida cotidiana y los valores durante la adolescencia afectan los resultados posteriores. Proponemos hacer esto por medio de la realización de un seguimiento de los participantes en el tiempo en al menos dos proyectos actuales e incorporar una estrategia longitudinal en los nuevos proyectos. Con el reconocimiento de que el desarrollo de valores a nivel individual debe llevarse a cabo en el contexto de un entorno favorable, los nuevos proyectos también prestarán más atención a los niños y hombres, con un enfoque en una estrategia comunitaria para la prevención de la violencia de género.

\section{Population Council}

El Population Council cambia la manera en la que el mundo piensa acerca de los problemas de salud y desarrollo. Buscamos entender las causas y las consecuencias de la desigualdad de género y las disparidades en las oportunidades que surgen durante la adolescencia. Brindamos las evidencias para desarrollar mejores programas y políticas prácticas que garanticen una transición exitosa y productiva hacia la edad adulta en los países en vías de desarrollo. www.popcouncil.org

(c) 2011 The Population Council, Inc.

4 - Visite www.popcouncil.org/publications/serialsbriefs/TABriefs.asp para obtener todos los resúmenes de la serie Promoting healthy, safe, and productive transitions to adulthood (Promoción de transiciones a la vida adulta sanas, seguras y productivas). 